\title{
Standardizing Care Coordination Within the Department of Veterans Affairs
}

\author{
Clinton L. Greenstone, MD 1,2,3,4, Jennifer Peppiatt, MSN, RN CNL' \\ Kristin Cunningham, $P M P^{7}$, Christina Hosenfeld, $M P H^{7}$, Michelle Lucatorto, DNP, FNP-C', \\ Michael Rubin, LCSW-C', and Adrienne Weede, LCSW-C ${ }^{l}$ \\ 'US Department of Veterans Affairs, Washington, DC, USA; ${ }^{2}$ VA Ann Arbor Health Care System, Ann Arbor, MI, USA; ${ }^{3}$ Department of Internal \\ Medicine, University of Michigan, Ann Arbor, MI, USA; ${ }^{4}$ Clinical Integration, VHA Office of Community Care, Washington, DC, USA.
}

J Gen Intern Med 34(Suppl 1):S4-S6

DOI: $10.1007 / \mathrm{s} 11606-019-04997-6$

(c) Society of General Internal Medicine (This is a U.S. government work and not under copyright protection in the U.S.; foreign copyright protection may apply) 2019

$\mathrm{T}$ he Veteran's Health Administration (VA) oversees the nation's largest integrated health care system, providing health care to approximately nine million Veterans at 1243 different health care facilities. ${ }^{1}$ VA provides health care that is similar or better in quality compared to non-VA health care systems. ${ }^{2}$ In the event that a Veteran is unable to receive timely care at a VA facility, VA provides the Veteran with the option of receiving care from a non-VA provider through VApurchased community care. Factors that currently may lead a Veteran to choose community care include a long wait time at his or her local VA facility, long geographic distance to VA providers for the care needed, or requiring care that VA does not provide (e.g., obstetrics). The VA MISSION Act of 2018, signed on June 6, 2018, established a permanent community care program for Veterans, increasing the options Veterans have for where and when they receive health care. ${ }^{3}$

VA schedules approximately 250,000 appointments a day within VA facilities, and approximately 102,000 a day for VApurchased community care. Nearly $34 \%$ of all VA outpatient appointments in fiscal year (FY) 2018 were provided in the community. Community care visits to both inpatient and outpatient facilities are usually for the entire episode of care for a specific need; for example, if a Veteran is seen in the community for a knee replacement, the episode of care might include appointments for a pre-operative appointment, x-ray, surgery, and post-operative physical therapy. Patients receiving community care will encounter a greater number of transitions of care compared with patients seen entirely within VA facilities, and these transitions could present potential risks to Veterans. ${ }^{4}$, ${ }^{5}$ These risks include, but are not limited to, missing community care appointments that are part of a complicated episode of care, not having critical information obtained in the community made available to VA providers to follow up upon, or

Published online May 16, 2019 receiving duplicative services from both VA and community providers that may be invasive and potentially dangerous. As compared to non-Veterans, Veterans have a greater number of comorbidities that need to be considered and well-coordinated when receiving health care. ${ }^{6-11}$

To address and mitigate these risks, VA has developed two initiatives aimed at helping Veterans navigate the intricacies of an increasingly complex and diverse provider network: the VA Office of Community Care (OCC) Care Coordination Model, and the collaborative Care Coordination and Integrated Case Management (CC\&ICM) Initiative. These initiatives stand up a framework by which internal VA case managers and care coordinators work together and with facility community care office care coordinators to ensure transitions of care inside VA and in the community are well-organized and focus on highrisk Veterans. These initiatives are summarized below.

\section{CARE COORDINATION MODEL IN VHA}

To prevent gaps in care, transitions in care both within and across health care systems require extensive planning and systematic support. The VA Office of Community Care (OCC) is developing a consolidated community care program with a standardized practice approach to ensure that VApurchased community care is coordinated through the entire episode of care and is seen as a critical component to support this effort. Within this practice model, standard care coordination processes will enable VA OCC to arrange services and resources based on Veterans' clinical needs, supporting timely access to community care followed by safe transitions of care back to VA. Within this model, a standardized tool assesses Veterans' risk for care coordination failures in the community. A standardized care coordination plan note is utilized, and new referral and authorization systems ensure that critical components of the care plan are followed up appropriately.

The VA OCC Care Coordination Model embraces Veterans' care needs in the community and is based on five key principles:

- Develop a personalized plan of care visible to all stakeholders and enable access to supporting medical documentation. 
- Create a mechanism for seamless transmission of data within the high-performing network of providers both internal and external to VA.

- Develop and maintain collaborative partnerships with VA- and community-based providers, care coordinators, care managers, and case managers who are part of the high-performing network.

- Ensure the high-performing network provides Veterans with high-quality and timely care.

- Develop a national and local governance structure with clearly defined roles, responsibilities, and measurable outcomes for care coordination.

For every Veteran referred to community care, the Care Coordination Model, aided by a Screening/Triage tool, determines a clinically indicated level of care coordination. There are four complexity levels: basic, moderate, complex/chronic, and urgent. The level of care coordination and risk status is based upon several social determinants of health, as well as risk of hospitalization or death within the next 1 year utilizing VA's Care Assessment Needs (CAN) score. The CAN score is a VA-developed predictive measure that includes new risk factors including age, emergency department and hospitalization histories, medical diagnoses, and laboratory studies validated against actual outcomes. Every VAMC has a facility Community Care Office staffed by an integrated team of clerical, administrative, and clinical staff. For every Veteran receiving community care, the predominant clinical, social, and timing factors that drive the level of care coordination complexity will help the OCC care coordinator develop the Veteran's personalized care plan.

Once created, the care plan is implemented by an interprofessional health care team including, but not limited to, a care coordinator, nurse, provider, administrative support assistant, and social worker. This team is responsible for following the Veteran through their community care-based episode of care to ensure dynamic, ongoing care facilitation. Additionally, VA is implementing a new electronic referral and authorization system that doubles as a portal for bidirectional exchange of health information with community providers. Utilizing this system, VA and community health care team members will have access to vital medical record information. Thoughtful use of that information can reduce service duplication and prevent potentially harmful medication interactions, thereby potentially improving Veteran safety. It is critically important to support the need for interoperability of health information using EHR systems to help facilitate timely information exchange in the care coordination process.

\section{PARTNERSHIP WITH OFFICE OF NURSING SERVICES/ OFFICE OF CARE MANAGEMENT AND SOCIAL WORK}

To maximize the effectiveness of its Care Coordination Model, VA OCC partnered with the VA Offices of Nursing Services and Care Management and Social Work, which launched a Care Coordination and Integrated Case Management (CC\&ICM) Initiative. Working together, the aim is to achieve a high-quality care coordination system across the Veterans health care continuum. The OCC Care Coordination Model and CC\&ICM Initiative provide complementary tools and processes that enhance VA's care coordination capabilities for Veterans accessing care provided in VA facilities and VA-purchased community care.

The CC\&ICM Initiative also adopts a Veterans whole health approach and encompasses the following core elements: Care Coordination Review Team (CCRT), a care stratification methodology, and a Lead Coordinator (LC). CCRTs comprise case managers supporting individual Veterans, care managers supporting a population of Veterans with a shared diagnosis, and care coordinators supporting Veterans across multiple care environments. As CCRTs become fully established at VAMCs, they will leverage the existing programs/areas across VA, such as primary care, specialty care, mental health, and community reintegration programs. This multiprogram approach fosters coordination across existing case and care management programs, respecting both a balance of expertise and the need for identification of a lead coordinator. ${ }^{12}$ These representatives report up through the appropriate chain of command for review of their work and work load, which ensures defined roles, responsibilities, and clinical outcomes.

The CCRTs ensure that high-risk Veterans are placed into the right level of intensity and type of care coordination services. The CCRT process uses a combination of triggers, including risk predictive tools, cost and service outlier reports, and internal referrals, to identify Veterans appropriate for review. These tools predict a Veteran's level of risk based on a variety of factors such as burden of medical disease, opioid overdose, homelessness, frailty, and suicide behavior. Existing disease-based registries, which are listings of Veterans with specific exposures or diseases, identify Veterans requiring specific services and those going through transitions in care.

The CCRTs utilize a care stratification methodology to stratify Veterans into the most appropriate level of facilitated care. These include care coordination, care management, and case management services. A Veteran's risk and acuity determines the intensity of interventions required by clinicians to promote their wellness and safety. Also, each of the case and care management services corresponds to a specific care coordination complexity level as a means for synchronizing care along a single continuum. At each level, Veterans are assigned to a Lead Coordinator via a CCRT. This assignment is based upon the level of complexity determining if a nurse or potential social worker would be most appropriate and the clinical service that is best suited to support the Veteran's most significant diagnostic need like cardiology, GI, or endocrinology. The Lead Coordinator serves as an easily identifiable and accessible point of contact for the Veteran and interprofessional health care team and oversees the Veteran's whole health care plan. The designation of a Lead Coordinator is to provide 
Veterans a single point of contact to support seamless care transitions, and should aid in increased Veteran engagement, reduced readmission rates, and improved clinical outcomes. The effectiveness of the Lead Coordinator is one of the components and outcomes of the initiative being assessed since no clear evidence supporting this model currently exists.

The health status of Veterans is dynamic, making Veteran identification and screening paramount to high-quality care coordination. Although most Veterans will be identified through a triggering mechanism or registry, CCRT referrals may be placed by clinicians involved in a Veteran's care anytime a Veteran's health status changes or a new care plan is needed. Through the CCRT, the OCC Care Coordination Model and CC\&ICM Initiative operationalize the same level of care coordination framework (i.e., basic, moderate, complex, and urgent) to support the establishment of a standardized complexity level for each Veteran, and ultimately, a VHA-wide care coordination system.

\section{CONCLUSION}

With the goals of focusing resources more effectively (e.g., reducing hospitalizations, readmissions, emergency department visits, and revisits) and improving the overall Veteran experience (e.g., safety, satisfaction, and health outcomes), the OCC Care Coordination Model and CC\&ICM Initiative are now being implemented at the VAMC in Salisbury, NC, in 2019. This initial implementation will generate feedback from the staff using the screening tools, standardized plan notes, and care coordination tools embedded in VA's new referral and authorization system. This information is critical to inform the national deployment of the care coordination and integrated case management practice going forward. Also, ongoing partnerships with several VA Health Services Research \& Development (HSR\&D) evaluation projects will assess access, quality, and costs of VA care coordination efforts and will provide evidence. As VA continues to transform its business processes and tools to improve Veterans' access, a successful care coordination model will remain at the heart of its success.
Corresponding Author: Clinton L. Greenstone, MD; Clinical Integration, VHA Office of Community Care, Washington, DC, USA (e-mail: Clinton.Greenstone@va.gov).

\section{REFERENCES}

1. Huang G, Kim S, Muz B, Gasper J. 2017 Survey of Veteran Enrollees' Health and Use of Health Care. April 2018. Available at: https://www.va. gov/HEALTHPOLICYPLANNING/SOE2017/VA_Enrollees_Report_Data_ Findings_Report2.pdf

2. Anhang Price R, Sloss EM, Cefalu M, Farmer CM, Hussey PS. Comparing quality of care in Veterans Affairs and non-Veterans Affairs settings. J Gen Intern Med 2018; 33: 1631-8.

3. John S. McCain III, Daniel K. Akaka, and Samuel R. Johnson VA Maintaining Internal Systems and Strengthening Integrated Outside Networks Act of 2018. Pub. L. No. 115-182. 2018. Available at: https:// www.congress.gov/115/bills/s2372/BILLS-115s2372enr.pdf

4. Rosen AK, Wagner TH, Pettey WB, Shwartz M, Chen Q, Lo J, O’Brien WJ, and Vanneman ME. Differences in Risk Scores of Veterans Receiving Community Care Purchased by the Veterans Health Administration. Health Serv Res 2018; 53: 5438-54.

5. Zuchowski JL, et al. Coordinating Care across Healthcare Systems for Veterans with Gynecologic Malignancies: A Qualitative Analysis. Med Care 2017; 55:S53-S60

6. Patel N, Golzy M, Nainani ND, Carter RL, and Lohr JW. Prevalence of various comorbidities among Veterans with chronic kidney disease and its comparison with other data sets. Ren Fail 2016; 38(2): 204-8

7. Agha Z, Lofgen RP, and VanRuiswyk JV. Are Patients at Veterans Affairs Medical Centers Sicker? A Comparative Analysis of Health Status and Medical Resource Use. Arch Intern Med 2000; 160(21): 3252-3257.

8. Kotagal V, Albin RL, Müller ML and Bohnen NI. Cardiovascular risk factor burden in veterans and non-veterans with Parkinson disease. J Park Dis 2018; 8(1):153-60.

9. Gray KE, Katon JG, Rillamas-Sun E, Bastian LA, Nelson KM, LaCroix AZ and Reiber GE. Association between chronic conditions and physical function among veteran and non-veteran women with diabetes. The Gerontologist 2016; 56(Suppl 1): S112-25.

10. Trivedi RB, Post EP, Sun H, Pomerantz A, Saxon AJ, Piette JD, Maynard C, Arnow B, Curtis I, Fihn SD and Nelson K. Prevalence, comorbidity, and prognosis of mental health among US veterans. Am J Public Health 2015; 105(12): 2564-9.

11. Ayers CR, Dozier ME, Pittman JO, Mayes TL and Twamley EW. Comparing clinical characteristics and treatment outcomes between Veterans and non-Veterans with hoarding disorder. Compr Psychiatry 2018; 86: 1-5.

12. Weaver SJ, Che XX, Perterson LA, and Hysong SJ. Unpacking Care Coordination Through a Multiteam System Lens: A Conceptual Framework and Systematic Review. Med Care 2018; 56(3): 247-259.

Publisher's Note Springer Nature remains neutral with regard to jurisdictional claims in published maps and institutional affiliations. 